\title{
Are the spatial patterns of weeds scale-invariant?
}

\author{
Roger Cousens, Jacco Wallinga and Michael Shaw
}

Cousens, R., Wallinga, J. and Shaw, M. 2004. Are the spatial patterns of weeds scaleinvariant? - Oikos 107: 251-264.

\begin{abstract}
In previous empirical and modelling studies of rare species and weeds, evidence of fractal behaviour has been found. We propose that weeds in modern agricultural systems may be managed close to critical population dynamic thresholds, below which their rates of increase will be negative and where scale-invariance may be expected as a consequence. We collected detailed spatial data on five contrasting species over a period of three years in a primarily arable field. Counts in $20 \times 20 \mathrm{~cm}$ contiguous quadrats, 225,000 in 1998 and 84,375 thereafter, could be re-structured into a wide range of larger quadrat sizes. These were analysed using three methods based on correlation sum, incidence and conditional incidence. We found non-trivial scale invariance for species occurring at low mean densities and where they were strongly aggregated. The fact that the scale-invariance was not found for widespread species occurring at higher densities suggests that the scaling in agricultural weed populations may, indeed, be related to critical phenomena.
\end{abstract}

R. D. Cousens, School of Resource Management, The Univ. of Melbourne, Victoria 3010, Australia.(rcousens@unimelb.edu.au).J. Wallinga, National Institute for Public Health and the Environment, P. O. Box 1, 3720 BA Bilthoven, the Netherlands. - M. W. Shaw, School of Plant Sciences, The University of Reading, Whiteknights, Reading RG6 6AS.

The description and interpretation of patterns in plant populations and communities have long been central themes in ecology. Patterns can be identified at scales from hundreds of kilometres (e.g. zonation due to climate) to centimetres or less (e.g. lichen mosaics on rocks). It has been traditional to study vegetation at a given site with a single quadrat size and arbitrary (usually regular) spacings between observations (Rew and Cousens 2001). Clearly, the way in which a pattern is perceived will depend on the degree to which scales of aggregation within the pattern and the scale of observation coincide (Dungan et al. 2002). Increasingly, ecologists have become interested in the ways in which measures of pattern of a species change across scale of observation (Kunin 1998). Are species that are highly aggregated at one scale necessarily aggregated at other scales? What do the relationships between type of pattern and scale of observation look like?
For many years, analysis of spatial pattern in plant populations was based primarily on indices related to the mean and variance at a single quadrat size (Morisita 1959, 1962, Southwood 1966, Lloyd 1967, Pielou 1977, Dessaint et al. 1991). It was noted that these indices varied with the size of the sampling unit and with the mean (Pielou 1957, Southwood 1966, Sawyer 1989, Richter and Söndgerath 1990, Wiles et al. 1992). Although, perhaps, considered as an annoying feature or a flaw of the methods, this was also an indication that information about scale was being missed. The introduction of statistical measures that explicitly address scaling phenomena has had a great impact on the analysis of spatial pattern. Many of these measures are closely inter-related and are concerned with the autocorrelation between spatial locations (Dale et al. 2002).

When spatial patterns are viewed at different scales, statistical measures of the pattern change in character-

\footnotetext{
Accepted 18 March 2004 
istic ways. It is common to find that relationships between some statistical measures and scale of observation follow power laws (straight lines on $\log -\log$ plots) over a wide range of scales and this observation has led to the development of fractal geometry (Mandelbrot 1982). Patterns were identified that appear the same regardless of the scale of observation, referred to as being self-similar or scale-invariant. A scale-invariant pattern can be detected if a $\log -\log$ plot of the measure against scale of observation produces a straight line; the slope of that line (i.e. the exponent in the "scaling" relation) has the interpretation of a dimension. A rather trivial example is a square. Its area varies with the square of its edge: plotting area as a measure versus edge as scale on a $\log -\log$ plot gives a straight line with slope 2 , and this indicates that we are studying a two-dimensional object. Non-trivial scaling occurs if the slope has a non-integer value: it is then called a fractal dimension. Such a dimension is constant over all scales and summarizes many of the properties of the pattern. There is a wide range of definitions of fractal dimension (indeed, an infinite number - Hentschel and Procaccia 1983), differing in the information that they provide, and an array of methods for their calculation (Grassberger and Procaccia 1983, Schroeder 1991). These are being applied increasingly to the analysis of biological structures, to spatial patterns in populations and communities, and to the physical environment in which the organisms live (Kunin 1998, Kunin et al. 2000, Enquist et al. 2002, Haskell et al. 2002).

Spatial patterns in plant populations are unlikely to be truly scale-invariant, as the abiotic environment of plants is not perfectly homogeneous in space and is subject to erratic events. Moreover, plant size will set a minimum range and the size of the mapped area will set a maximum range for scaling. As a result, patterns may approximate scale-invariance only over a certain range of scales. If this range is small, (differing, say, by a factor of ten - i.e. one order of magnitude) it is hardly meaningful to talk about scale-invariance, and the fractal dimension is not an informative feature (Avnir et al. 1998). Scaling behaviour over a wide range of scales, with constant fractal dimension, has been found in patterns of some plant populations. For example, Kunin (1998) found evidence for scale-invariance in Gladiolus illyricus and Lathyrus japonica over at least two orders of magnitude of scale. Wallinga (1995) and Wallinga et al. (1998) also found that Galium aparine in a $18 \times 32.4 \mathrm{~m}$ area was scale-invariant at scales from 0.2 to $10 \mathrm{~m}$.

It is, perhaps, difficult to envisage why plant patterns should exhibit scale-invariance. There are a range of processes that lead to either greater or lesser aggregation, each of these acting at a distinct characteristic scale. For example, passive dehiscence may limit seed dispersal of annual plants to within tens of centimetres, dispersal by ants may be limited to a few metres, while machinery will spread seeds much greater distances (although some seeds may be spread over very long distances, the mean distance may remain short; Howard et al. 1991, Rew and Cussans 1997). Spatial variation in fecundity will occur over distances of centimetres as a result of competition with other species and herbivory, or over much greater distances as a result of soil type, topography and hydrology. Repeated disturbance of plant populations, through falling trees or trampling, often having effects that are unpredictable in size and location, tend to leave their marks at particular scales. Each of these processes with a characteristic scale suggests formation of particular types of pattern dominating at particular scales, not scale-invariance.

There is, however, a theoretical rationale for expecting scale-invariance in at least some types of plant populations. Notably, it has been found in various fields of science that power laws and scale-invariance are likely to occur when a system remains close to a so-called critical threshold (Sole et al. 1999, Levin 2003). For organisms, the critical population dynamic threshold occurs when, on average, births and deaths just balance. The long-run fate of a population close to this threshold then exhibits divergent behaviour in the sense of Shaw (1995): in repeated colonisations of space, the species will sometimes become extinct, sometimes become more common. This will be true of every demographic lineage at every location, so that there will be gaps and patterns on every scale (Shaw 1994).

Such divergent behaviour will be particularly apparent where the species barely persists at very low abundance (Grassberger 1983). We might therefore expect endangered species to have the property of scale-invariance. Also, populations of generally common species may exhibit such a phenomenon in specific circumstances where their overall density is kept low by human intervention (note that even a species occurring at a low average density may occur locally at very high densities if it is especially patchy). For example, Wallinga (1995) and Bröker and Grassberger (1998) found that the output from an individual-based spatial simulation model of an annual plant population was scale-invariant over at least three orders of magnitude, if its overall abundance was kept at low levels. Bröker and Grassberger (1998) showed that this phenomenon can be categorised as so-called "self-organised criticality". An example of a population kept at low density is a weed in an agricultural field: although weeds may be numerous, their abundance would be far greater in the absence of herbicides and cultivation. Population models for some common weeds have predicted that the critical mortality required for populations to decline to zero is within the range found for modern herbicides (Cousens et al. 1986, Doyle et al. 1986). Demography, alone, in an otherwise homogeneous environment, may generate patchiness 
over many scales in simulation models (Wallinga 1995). It has thus been hypothesised that for weed populations at low densities, scaling might be a general phenomenon (Wallinga 1995).

The first step in examining the generality of this hypothesis is to examine whether non-trivial scaling occurs, and if so, over what range, for a wider range of weed species with contrasting ecologies. A second step is to examine how the scaling range varies for different overall densities: if we find wide scaling ranges also for weed species occurring at high overall densities, it would lead to rejection of the hypothesis that scaling is due to self-organised criticality. Although there has been a proliferation of maps of weeds in recent years, few of these have examined a sufficient range of scales to allow fractal dimensions to be calculated. Most weed data are collected from quadrats spaced out on a grid (Rew and Cousens 2001), resulting in few observations at a limited range of scales. In this paper, we present analyses of a highly detailed data-set, collected expressly for the purpose of examining the influence of scale of observation on apparent spatial patterns of weeds. We use three methods to examine whether patterns show scale-invariance and the extent to which the scaling relationships vary among species of contrasting biology and over time.

\section{Methods}

\section{Study site}

A 0.9 ha area within a field in the Wimmera region of Victoria, Australia was surveyed three times, in alternate years. The field had been managed for the previous 10-15 years in a rotation of subterranean clover (Trifolium subterraneum L.) based pasture and wheat (Triticum aestivum), although in recent years the farmer has diversified the crop sequence and only seldom includes pasture. The soil is a sandy loam, with gravel and larger stones in some patches. Crops grown over the study period were wheat, undersown with sub-terranean clover (1998), self-regenerating pasture (1999), canola (Brassica napus) (2000), wheat (2001) and barley (Hordeum vulgare) (2002). Further details of the site, along with management details for 1998, are given by Cousens et al. (2002).

Fourteen species were recorded in the three years of mapping. Some, such as Lamium amplexicaule (L.), Malva parviflora L., Avena fatua L., Acetosella vulgaris Fourr. sens.lat. and Cirsium vulgare (Sav.) Ten. were at very low densities and would not have provided analysable data. Lolium rigidum Gaud. and Crassula spp. were so abundant that an excessive time would have been needed for mapping, while Avena fatua (and possibly Avena sterilis ssp. ludoviciana (Durieu) Nyman) were difficult to identify among the cereal plants. Detailed maps were therefore produced for Erodium botrys (Cav.)
Bertol., Erodium cicutarium (L.) L'Her., Arctotheca calendula (L.) Levyns, Buglossoides arvense (L.) I. M. Johnson, and Oxalis pes-caprae L. Of these five species only $B$. arvense seeds are believed to be taken up into the harvester and dispersed in that way; $O$. pes-caprae reproduces only vegetatively and is thus mainly spread by cultivation; and E. botrys and E. cicutarium disperse seeds by explosive discharge from the pod.

\section{Survey design}

Surveying took place after the primary emergence event, so that few new weed plants were appearing. This depended on the sowing date of the crop. Counts were made from 22-26 June 1998, 19-23 June 2000 and 29 July - 1 August 2002. The area was marked out with string into $5 \mathrm{~m}$ wide strips. A rigid frame, $5 \mathrm{~m}$ wide and divided into two rows of twenty-five $20 \times 20 \mathrm{~cm}$ quadrats, was placed across a strip for counting, then moved forward $40 \mathrm{~cm}$ after plants were counted. Five observers recorded the number of plants in blocks of ten quadrats along the frame; recorders changed position after each strip. Counting took five days, during which time there was virtually no further emergence. This size of quadrat was selected so that it was small enough to derive a wide range of larger quadrat sizes from the data and yet large enough so that edge effects and errors in quadrat placement were not too great. It is also roughly the size of a single rosette of $A$. calendula or Erodium spp. The entire area was mapped in 1998, while about half and two thirds were mapped in 2000 and 2002 respectively.

\section{Data analysis}

Data were entered into a spreadsheet and a Fortran program was used to combine the original quadrat counts into larger quadrats of a given size and shape. A square of $450 \times 450$ quadrats $(0.81 \mathrm{ha})$ at the northern-most end of the area was analysed for 1998, allowing the construction of square quadrats of side $0.2,0.4,0.6$, $1.0,1.2,1.8,2.0,3.0,3.6,5,6,9,10,15,18,45$, and $90 \mathrm{~m}$, in all cases filling the complete area. The area in common for all three years was a rectangle $45 \times 75 \mathrm{~m}$ stretching from the northwest corner of that mapped in 1998. This could be completely filled by square quadrats with sides $0.2,0.6,1,3,5$, and $15 \mathrm{~m}$.

Several measures of counts within quadrats are available that allow us to find the scaling range, and if present, the fractal dimension. We used three:

\section{Correlation sum}

Divide the study area into rectangular quadrats of width $\times$ metres. If there are $\mathrm{N}$ plants in the mapped area and $n_{i}$ in quadrat $i$, the correlation sum is defined as 
$\mathrm{C}_{\mathrm{x}}=\sum \mathrm{p}_{\mathrm{i}}^{2}$

where $p_{i}$ is the proportion of the individuals that are present in quadrat $i$ (i.e. $n_{i} / N$ ). If $C_{x}$ against quadrat width on a $\log -\log$ plot forms a straight line (Fig. 1), the slope is the correlation dimension, $\mathrm{D}_{2}$ (Grassberger and Procaccia 1983, Wallinga 1995).

There are theoretical limits associated with $\mathrm{C}_{\mathrm{x}}$, and hence for $\mathrm{D}_{2}$. If plants are evenly spread across all quadrats, $D_{2}=2$ (the mean density is present in every quadrat, and $C_{x}=1 / Q$ where $Q$ is the number of quadrats of side $\mathrm{x}$ ). If all individuals are in one quadrat of the smallest size observed, $D_{2}=0\left(C_{x}=1\right.$ for all quadrat sizes); as quadrat size approaches plant size, single plants appear as single-dimensional points, and $\mathrm{D}_{2}=0$. It is also possible to calculate expected values for particular types of pattern. For example, ecologists often examine evidence for fit to a Poisson distribution and interpret this in relation to randomness. We therefore compared our observed $\mathrm{C}_{\mathrm{x}}$ data with a pattern that is random at all scales. At each quadrat size, we calculated expected frequencies of numbers in quadrats for a Poisson distribution with that mean plant density (Sokal and Rohlf 1995), and then calculated the corresponding value of $\mathrm{C}_{\mathrm{x}}$. We only did this for the full data-set (Fig. 4), as the Poisson distribution always appeared close to the lower theoretical boundary on a $\log -\log$ graph.

Statisticians will note that the quantity $\Sigma p_{i}^{2}$ is simply summing over the correlation function (hence the name correlation sum), and that is closely related to the Kfunction (Ripley 1981, Cressie 1991). Ecologists will note that the quantity $\Sigma \mathrm{p}_{\mathrm{i}}^{2}$ is analogous to Simpson's diversity

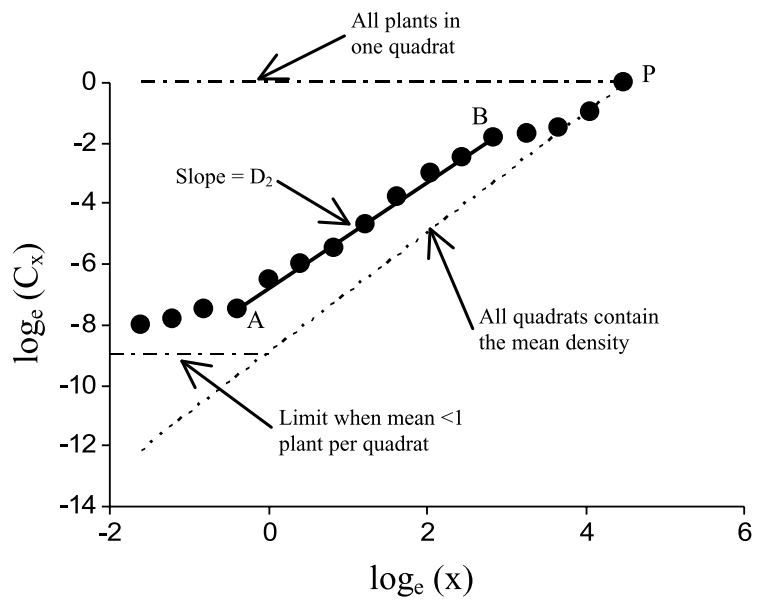

Fig. 1. Hypothetical empirical relationship between $C_{x}$ and $x$ on a $\log -\log$ plot, in which there is a section $\mathrm{A}-\mathrm{B}$ where the relationship is approximately linear. Such a linear section implies scale invariance over that range of scales. The dashed lines show theoretical boundaries beyond which combinations of $\mathrm{C}_{\mathrm{x}}$ and $\mathrm{x}$ cannot exist; the reasons for these boundaries are as labelled. Point $\mathrm{P}$ is where there is a single quadrat, so that completely even and maximum aggregation situations are indistinguishable. index (Pielou 1977), which uses the proportions of individuals in different species within a sample, rather than the proportions of individuals of a single species within different quadrats. Hence, in ecological parlance $\mathrm{C}_{\mathrm{x}}$ can be said to measure the diversity of counts among quadrats.

\section{Incidence}

In a very similar way to the procedure for the correlation sum, it is possible to use incidence, the number of quadrats where plants are present $\left(\Sigma \mathrm{p}_{\mathrm{i}}^{0}\right)$ to calculate the Haussdorf or box-counting dimension, $\mathrm{D}_{0}$ (Grassberger and Procaccia 1983, Schroeder 1991). For a $\log -\log$ plot of the reciprocal of the number of quadrats containing plants against quadrat width, the slope will be $\mathrm{D}_{0}$.

\section{Conditional incidence}

This method, proposed by Shaw (1996), examines the extent to which occupancy of an area at one scale is duplicated at the next scale down. The study area is first divided into a number of equal sections. The sections containing at least one individual are then each subdivided into the same number of sub-sections, and the total number of sub-sections containing individuals is recorded. Thus we can calculate the conditional incidence $\left(I_{c}\right)$ : given that an area contains at least one individual, what is the probability that an area at the next level of sub-division will contain at least one individual? This can be repeated at successively smaller divisions. Shaw (1995) originally used this method for a square area of $2^{\mathrm{n}}$ quadrats, dividing into quarters at each successive scale. Here, we again divided successively into quarters, but we used a slightly different algorithm to allow for a rectangular area (Appendix). The maximum value of $I_{c}$ will be 1 and, for sub-divisions into quarters, its minimum will be 0.25 .

Conditional incidence has an easy-to-visualise meaning directly related to what would be seen looking at presence/absence in the field with a resolution of a given scale. If the pattern of incidence is scale-invariant, then a graph of conditional incidence against scale will produce a horizontal line. However, as with the other methods, the graph can also be interpreted if the pattern is not strictly self-similar, since it will contain information about pattern at all scales examined. Visual patchiness corresponds to areas with more filled cells than usual; this will cause a peak in the conditional incidence. The scale at which there is greatest patchiness is the scale immediately greater than the peak in the conditional incidence. At ranges of scale where conditional incidence is constant, the relationship

$\mathrm{D}_{0}=2+\frac{1}{2} \log _{2}\left(\mathrm{I}_{\mathrm{c}}\right)$

holds. 


\section{Results}

Arctotheca calendula, the most abundant species, was spread throughout the field, although there were areas in which it was more concentrated than in others (Cousens et al. 2002). It also displayed anisotropy, with bands of high and low density running north-south (Fig. 2). Its abundance increased from 1998 to 2000, then (along with all other species) decreased in 2002 (Fig. 3). Areas of low density were more conspicuous in these later years. E. botrys was more abundant in the eastern half of the area (though never as abundant as $A$. calendula), and with a ridge of high density also reaching across to the western side in the centre of the area. It was spread thinly across these areas, rather than forming any clear patches. E. cicutarium was also spread thinly in 2000, mostly on the western side of the area. No plants were found in 1998 and very few in 2002. O. pes-caprae formed four very dense, comet-like patches, close together near the western edge. B. arvense formed loose clusters in various parts of the area, as well as having isolated individuals. No plants were observed in 2000 and there were so few in 2002 that analysis was not worthwhile. Total numbers of plants are given in Table 1. The mean densities tended to be highest for A. calendula, and decreasing in the order of $O$. pes-caprae, E. botrys, $E$. cicutarium to the lowest densities observed for B. arvense.

\section{Correlation sum}

All four species mapped in 1998 showed scaling behaviour for a wide range of quadrat sizes, i.e. the $\log -\log$ relationship between $\mathrm{C}_{\mathrm{x}}$ and $\mathrm{x}$ was linear (Fig. 4). A. calendula (the species at the highest mean density) (a)

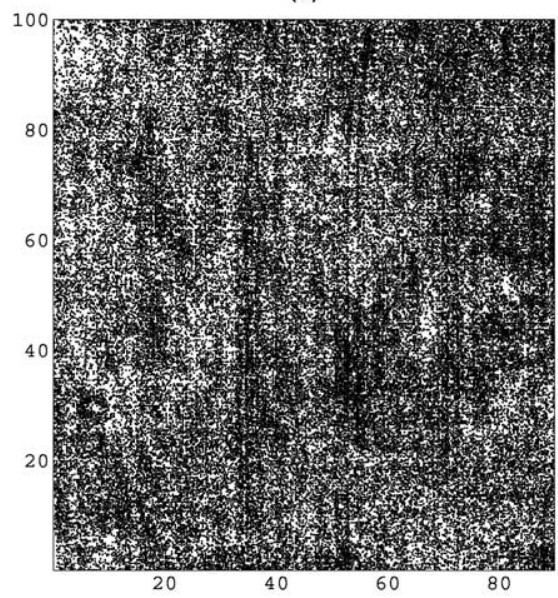

(b)

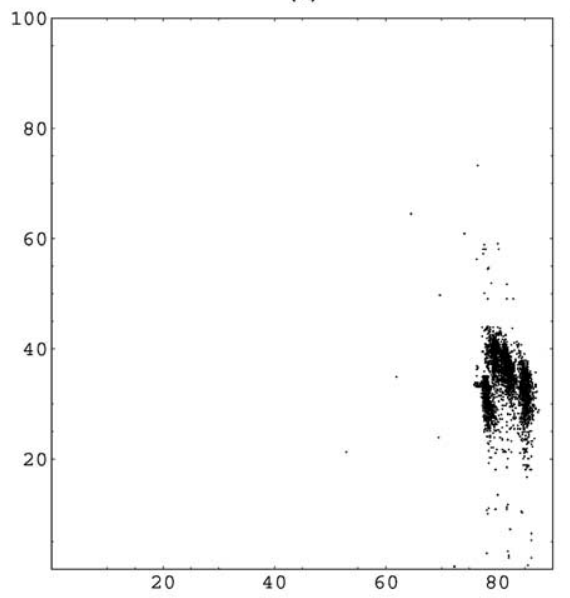

(c)

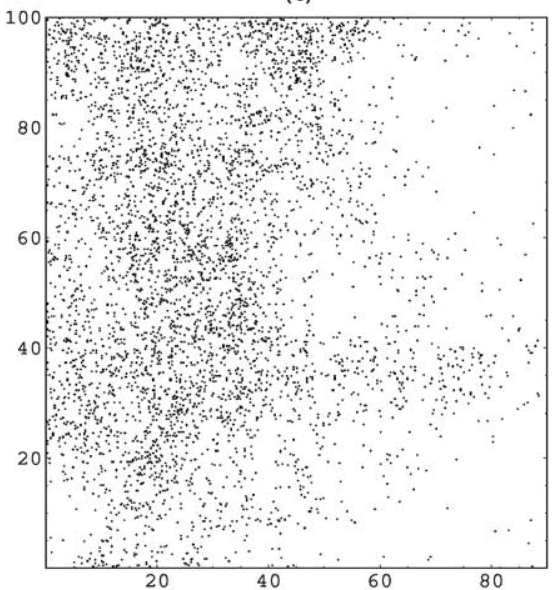

(d)

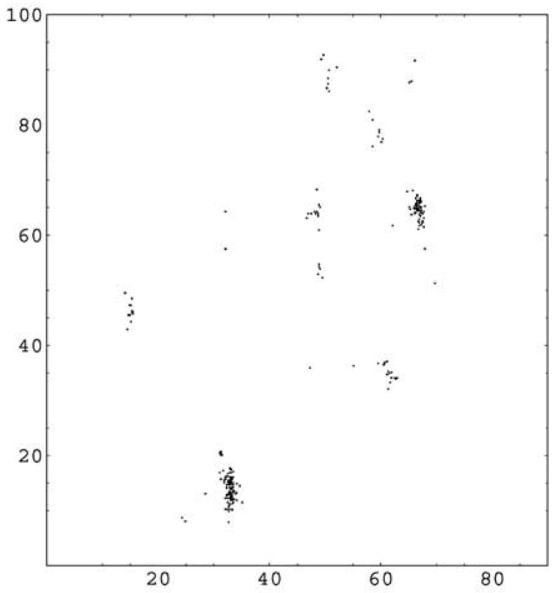

Fig. 2. Maps of presence/absence in $20 \times 20 \mathrm{~cm}$ quadrats for the four species counted in the $90 \times 90 \mathrm{~m}$ area in 1998. (a) A. calendula, (b) O. pes-caprae, (c) E. botrys, (d) B. arvense. Note that south is at the top. 
1998

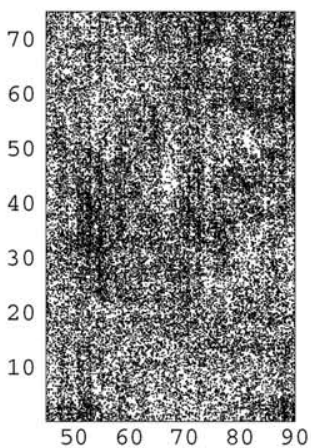

(b)

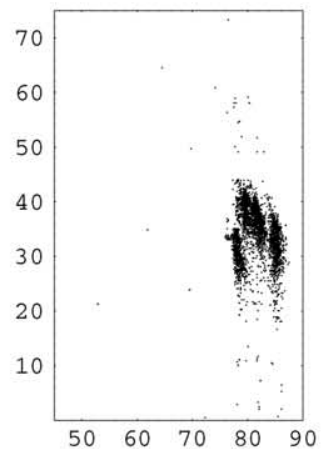

(c)

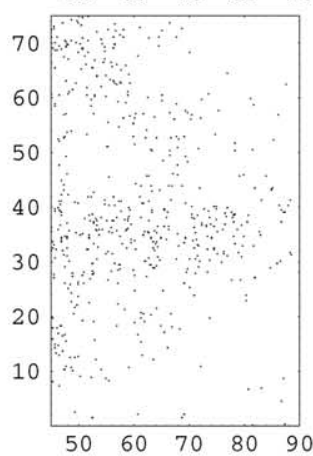

(d)

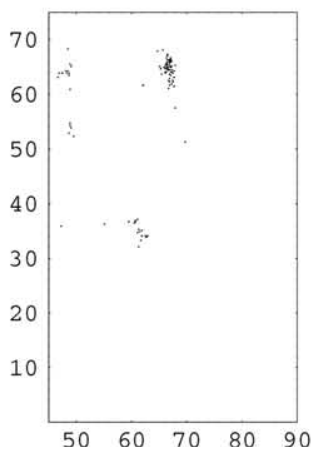

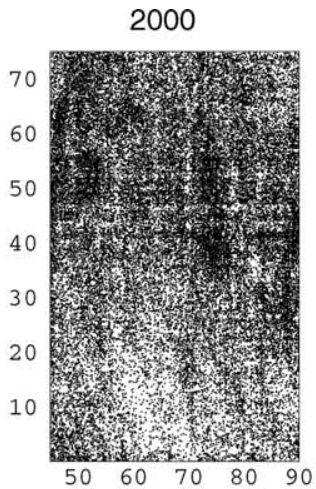
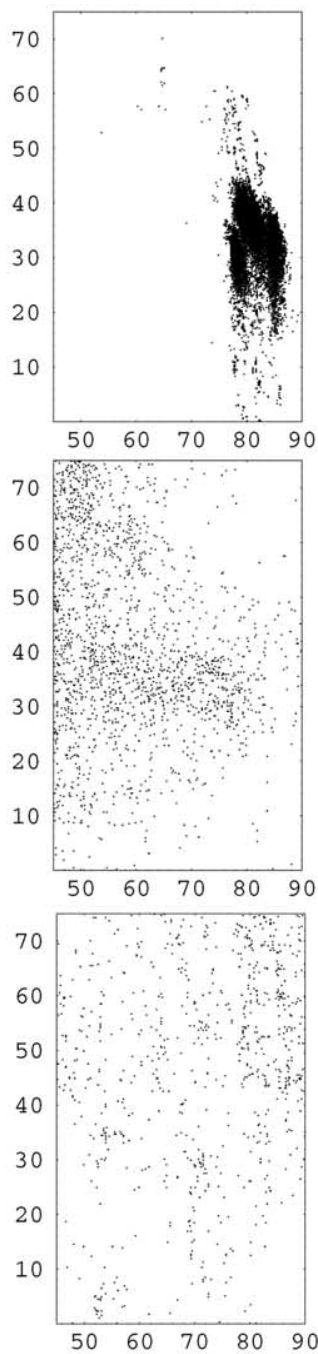
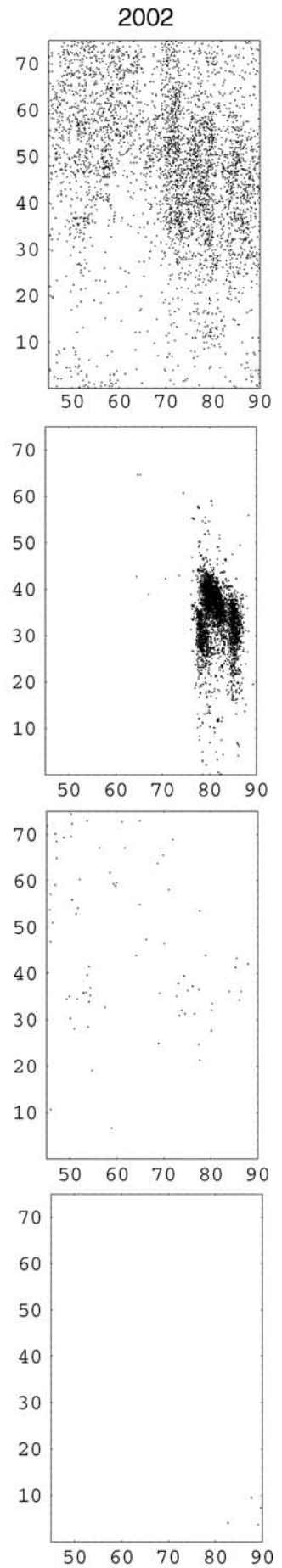

Fig. 3. Maps of presence/absence in $20 \times 20 \mathrm{~cm}$ quadrats for five species counted in the $75 \times 45 \mathrm{~m}$ area in all three years. Rows indicate (a) A. calendula, (b) $O$. pes-caprae, (c) E. botrys. In row (d) the 1998 graph is for $B$. arvense, the 2000 and 2002 graphs are for E. cicutarium . Note that south is at the top. showed trivial scaling behaviour with a value of the correlation dimension approximating 2 (Table 2), indicating the plant pattern is almost plane-filling.

$O$. pes-caprae and $B$. arvense, the two most patchy species at intermediate and low overall densities, showed non-trivial scaling behaviour over a wide range of scales. There appear to be two different scaling regions, with the slope of the $\log -\log$ plot of correlation sum versus scale decreasing at larger scales. The slopes of the two sections of each species were estimated using a linear spline 
Table 1. Numbers of plants of the various species recorded in the $90 \times 90 \mathrm{~m}$ mapped area in 1998 , or in the $75 \times 45 \mathrm{~m}$ mapped in 1998, 2000 and 2002.

\begin{tabular}{|c|c|c|c|c|c|}
\hline & A. calendula & E. botrys & E. cicutarium & O. pes-caprae & B. arvense \\
\hline \multicolumn{6}{|c|}{$90 \times 90 \mathrm{~m}$ area } \\
\hline 1998 & 105,085 & 4,774 & 0 & 5,701 & 289 \\
\hline \multicolumn{6}{|c|}{$75 \times 45 \mathrm{~m}$ area } \\
\hline 1998 & 43,060 & 554 & 0 & 5,701 & 111 \\
\hline 2000 & 50,936 & 1,860 & 869 & 33,474 & 0 \\
\hline 2002 & 4,149 & 78 & 4 & 11,243 & 1 \\
\hline
\end{tabular}

"broken stick" regression (Ross 1987) omitting the largest quadrat size. The change in slope occurred at $6.3 \mathrm{~m}(95 \%$ confidence intervals $2.7 \mathrm{~m}$ to $14.7 \mathrm{~m})$ for $O$. pes-caprae and at $6.7 \mathrm{~m}(95 \%$ confidence intervals $3.9 \mathrm{~m}$ to $9.8 \mathrm{~m}$ ) for $B$. arvense (large CI's are due to the logtransformation).

There are fewer quadrat sizes for the reduced area that was mapped in all three years (Fig. 5). Hence, there is poorer definition of linear sections and thus poorer estimation of the slope. Values of correlation dimension varied only slightly between years (Table 2). As in 1998, A. calendula appeared scale-invariant in both 2000 and 2002. The correlation dimension was somewhat lower in 2002, the year in which mean plant density was considerably reduced. With this range of quadrat sizes, O. pes-caprae appeared scale-invariant with a fractal dimension of 1.5. The dimension was somewhat larger when the plant abundance was highest and somewhat lower in the year when abundance was lowest. There were too few plants of $E$. botrys all years, and of $E$. cicutarium in 2000, to estimate slopes for these species.

\section{Incidence}

Again, all four species showed scaling behaviour in 1998, with the $\log -\log$ relationship between 1 /incidence and $\mathrm{x}$ being linear over a wide range of quadrat sizes (Fig. 6). Both E. botrys and A. calendula showed trivial scaling, over all but the smallest quadrat sizes, with a fractal dimension $\left(\mathrm{D}_{0}\right)$ very close to 2 indicating that species were almost always present at every scale. The graph for O. pes-caprae approximated to a straight line across all quadrat sizes, with a fractal dimension of 1.23 indicating that the species is aggregated at all of the observed scales. Unlike the correlation sum, incidence only indicated one
Fig. 4. Relationships between $\mathrm{C}_{\mathrm{x}}$ and $\mathrm{x}$ (logged axes) for the $90 \times 90 \mathrm{~m}$ area in 1998: (a) A. calendula, (b) O. pes-caprae, (c) E. botrys, (d) B. arvense. Regression lines (bold) were fitted to those parts of the relationship that appear to be approximately linear (see text for details); their slopes $\left(D_{2}\right)$ are given in Table 2. Dashed lines are theoretical limits (Fig. 1). Thin solid lines show calculated values for a Poisson distribution with a mean the same as the observed data.
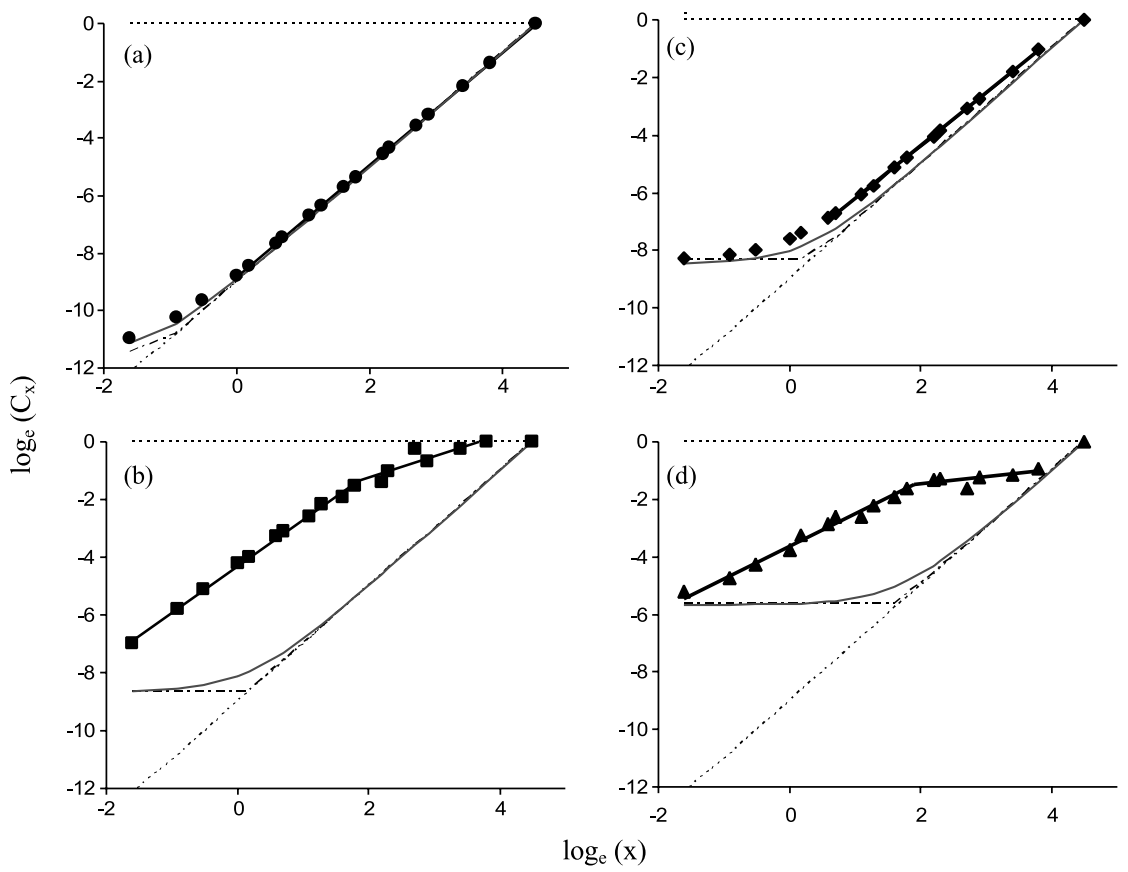
Table 2. Estimated fractal dimensions for the various species recorded in a $90 \times 90 \mathrm{~m}$ area in 1998 , or in the $75 \times 45 \mathrm{~m}$ area mapped in 1998, 2000 and 2002. Only those species are included for which there were sufficient numbers of plants to do a meaningful analysis. $\mathrm{D}_{0}$ is the fractal dimension calculated from incidence (the box-counting method), whereas $\mathrm{D}_{2}$ is calculated from the correlation sum. Two values of $\mathrm{D}_{2}$ are given where a broken-stick model seemed more appropriate than a straight line.

\begin{tabular}{lll}
\hline & $\mathrm{D}_{0}$ & $\mathrm{D}_{2}$ \\
\hline $90 \times 90 \mathrm{~m}$ area & & \\
1998 & & \\
A. calendula & 1.98 & 1.95 \\
O. pes-caprae & 1.23 & $1.59,0.86$ \\
E. botrys & 1.93 & 1.84 \\
$\quad$ B. arvense & 0.68 & $1.13,0.25$ \\
$75 \times 45 \mathrm{~m}$ area & & \\
1998 & 1.99 & 1.93 \\
A. calendula & 1.21 & 1.54 \\
O. pes-caprae & & \\
2000 & 1.99 & 1.93 \\
A. calendula & 1.42 & 1.68 \\
O. pes-caprae & & \\
2002 . calendula & 1.95 & 1.74 \\
O. pes-caprae & 1.40 & 1.59 \\
\hline
\end{tabular}

scaling region. The remaining species, $B$. arvense, appeared scale-invariant at all but the largest quadrat sizes, with a fractal dimension of 0.68 .

For the $75 \times 45 \mathrm{~m}$ area, the graphs for $\mathrm{D}_{0}$ in 1998 , 2000 and 2002 are qualitatively very similar to $D_{2}$, and are therefore not shown. The estimated fractal dimensions again increased with mean density (Table 2). Whereas $\mathrm{D}_{0}$ was slightly greater than $\mathrm{D}_{2}$ for $A$. calendula, the reverse was true for $O$. pes-caprae.

\section{Conditional incidence}

In 1998, A calendula had a constant $\mathrm{I}_{\mathrm{c}}$ for quadrats wider than $0.8 \mathrm{~m}$ (Fig. 7). This reflects the fact that the species is sufficiently evenly spread over the area that it is present in all quadrats above that size. $O$. pes-caprae also had a roughly constant $I_{c}$ over most scales, though with a slight reduction at intermediate scales. In quadrats where the plants occurred, an average of $60 \%$ of subdivided quadrats contained plants, regardless of scale. Three species, A. calendula, E. botrys and $B$. arvense had very low values of $I_{c}$ for small quadrat sizes, but much greater values for large quadrats. In the latter case, there was considerable scatter in the relationship.

Graphs for the smaller area observed in all three years (Fig. 8) are broadly similar to those for the full area in 1998. Values for very large quadrat sizes can show considerable differences between the full and reduced data-sets where the species is restricted to one part of the field, as in the case of $O$. pes-caprae. This simply reflects the restricted sampling: at the extreme, a single field cannot be used as evidence about variation between fields. A. calendula had $\mathrm{I}_{\mathrm{c}}$ of 1 at large quadrat sizes, being abundant and fairly evenly spread across the field. This is trivial scale-invariance, with $\mathrm{D}_{0}=2$ indicating simply that the plant is uniformly present. One species, $O$. pes-caprae, showed non-trivial scale invariance with an intermediate $I_{c}$ over almost all scales. All species had lowest values of $I_{c}$ at small quadrat sizes-close to the minimum possible for all but $A$. calendula and $O$. pescaprae-suggesting a definite spacing mechanism.

\section{Discussion}

The spatial patterns of the weeds that were either most aggregated and/or at the lowest overall densities in this study showed non-trivial scale-invariance, with fractal dimensions over a wide range of scales. Thus, the results clearly support the previous observation (Wallinga 1995) that scaling occurs in weed spatial patterns. The fact that species at the highest overall densities had trivial scaling behaviour with a fractal dimension approximating 2 indirectly supports the hypothesis that non-trivial scaling in homogeneous environments may be related to occurrence at very low overall densities, near so-called critical thresholds. Trivial scaling with a dimension of 2 at high densities merely indicates that the spatial plant pattern is space-filling, and no other specific aspects are detected (the pattern could be random or regular). For all four species, correlation sums expected for a Poisson distribution were very close to the minimum possible values (indicated by dashed lines in the figures). Thus it is difficult to distinguish between a random and a regular distribution using this method, and hence non-trivial scaling is only likely to be concluded where there is strong aggregation for whatever reason (biological or physical).

The observed scaling range depends on the method of analysis. Each method examines different properties of pattern. The correlation dimension, as used in Wallinga's (1995) original study, examines diversity of counts among quadrats and suggests scaling over one to two orders of magnitude, with this range being constrained at the upper limit by the size of the study area. The correlation sum and incidence approaches are insensitive to noise, because of the double-log nature of the plots used. This is particularly important for these data, which have a range of only two orders of magnitude of quadrat size. Two species, namely those that were most clearly patchy in their distribution, have changes in $\mathrm{D}_{2}$ at about $6 \mathrm{~m}$ for the 1998 data; results are less clear for other years, where there are too few points.

There are few published values with which to compare our estimates of fractal dimension. The value for high densities are near their theoretical maximum value of 
(a)
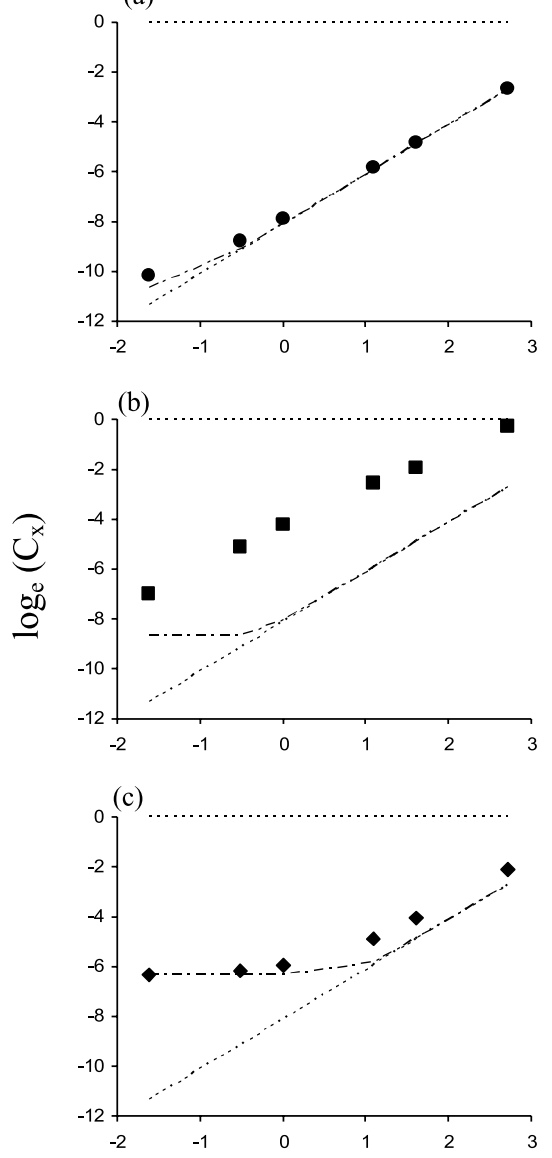

2000
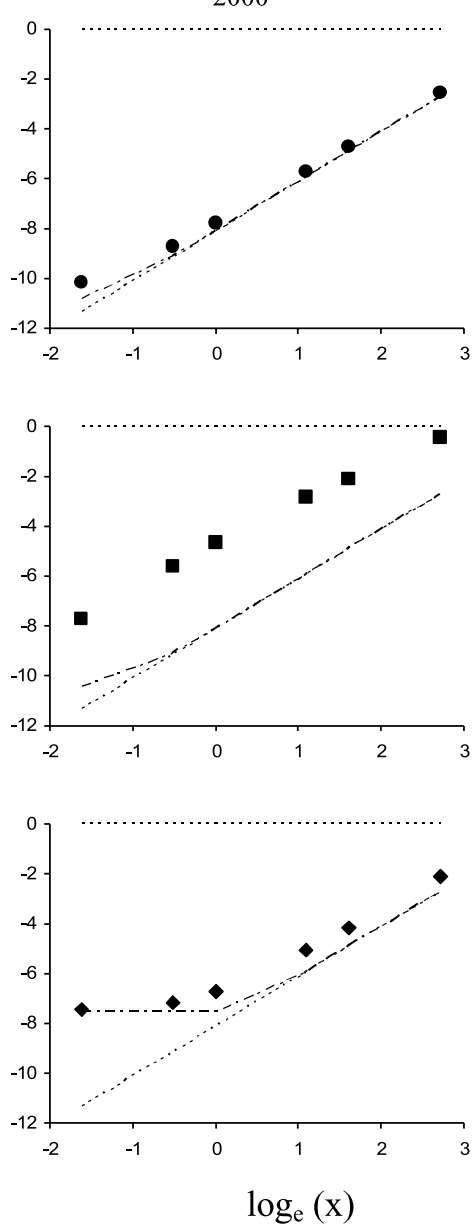

2002

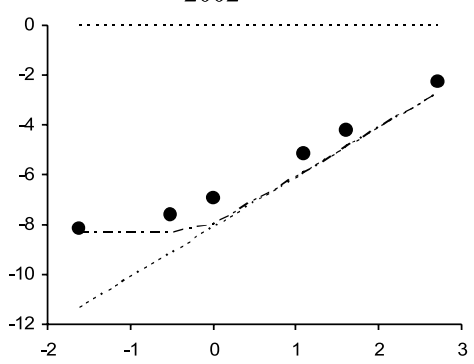

Fig. 5. Relationships between $\mathrm{C}_{\mathrm{x}}$ and $\mathrm{x}$ (logged axes) for the $75 \times 45 \mathrm{~m}$ area mapped in all three years: rows indicate (a) $A$. calendula, (b) O. pes-caprae, (c) E. botrys. Fractal dimensions $\left(\mathrm{D}_{2}\right)$ were calculated for those parts of the relationship that appear to be approximately linear (see text for details); their slopes are given in Table 2. Dashed lines are theoretical limits (Fig. 1).

$\mathrm{D}=2$. High values of $\mathrm{D}_{2}$ for $A$. calendula and $E$. botrys reflect the fact that these species, although at very different densities, are spread diffusely throughout the study area. Values for other species occurring at intermediate and low densities are near the value of $\mathrm{D}_{2}=1.51$ reported by Wallinga (1995) for G. aparine and the value of $\mathrm{D}_{2}=1.17$ that he reported for a model population. Low values of $\mathrm{D}_{2}$ for $O$. pes-caprae and $B$. arvense at larger scales indicate that they are aggregated in discrete parts of the area. There are some reports of fractal dimensions obtained using the widely-quoted method of Burrough (1983), such as 1.51-1.84 from the study of Leduc et al. (1994), but the lines in semivariograms are seldom straight enough, or over a wide enough range of scales, to conclude that patterns are scale-invariant.

There was only minor variation in the values of fractal dimensions among years, despite considerable changes in mean density and, particularly for A. calendula in 2002, in spatial locations of high and low densities. Some of the most dense areas in 1998 produced few seedlings in 2002, while other areas remained at a relatively high density. This indicates that, once a field has been occupied for some time, fractal dimensions and scaleinvariant relationships may be characteristic of species and habitats, rather than being dynamic variables responding to random events. Alternatively, it may be that they only vary over long time spans, at least in relation to the longevity of the seed bank. It would be interesting to see how scaling relationships vary over a wider range of species biologies: we might perhaps expect different behaviour in annuals vs perennials, in those species with greater vs lesser rates of population increase, and in species closely linked to edaphic conditions vs species that appear equally suited to conditions throughout fields. It would also be appropriate to examine the theoretical dynamics of pattern measures in relation to scale in hypothetical species, as they invade and then approach equilibrium within a homogeneous 

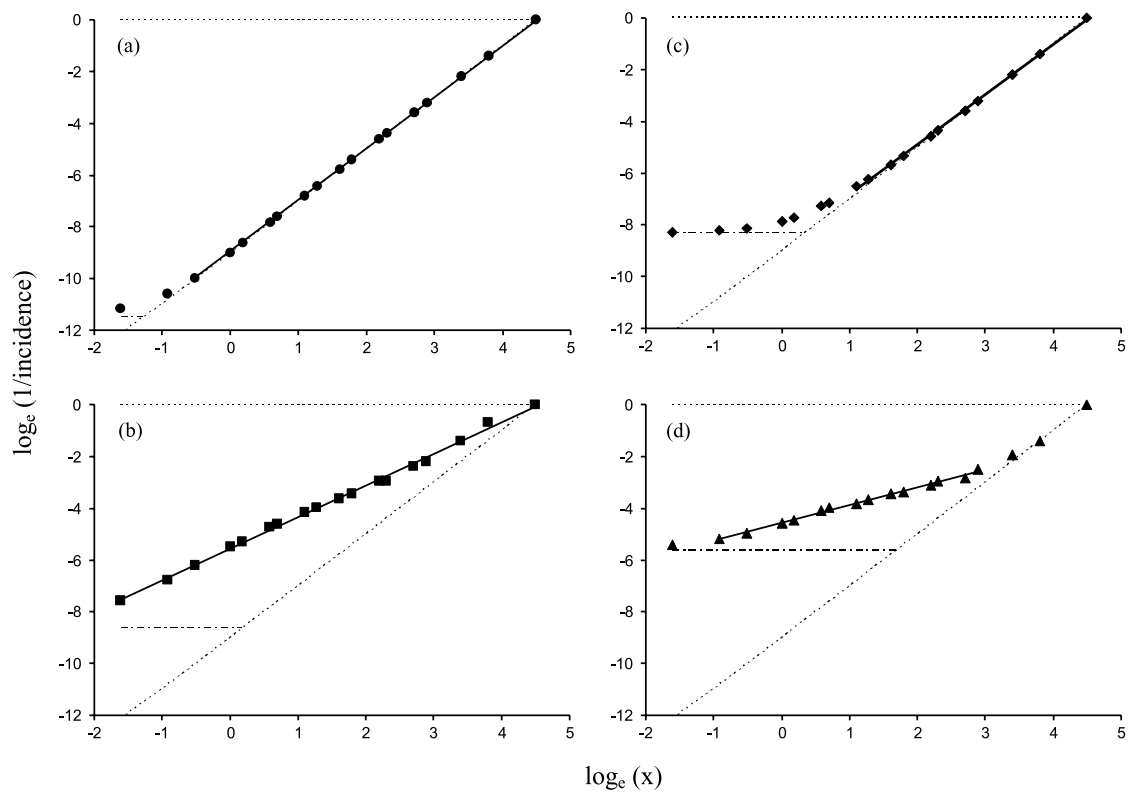

Fig. 6. Relationships between $1 /$ incidence and $\mathrm{x}$ (logged axes) for the $90 \times 90 \mathrm{~m}$ area mapped in 1998: (a) A. calendula, (b) $O$ pes-caprae, (c) E. botrys, (d) $B$. arvense. Regression lines (bold) were fitted to those parts of the relationship that appear to be approximately linear (see text for details); their slopes $\left(\mathrm{D}_{0}\right)$ are given in Table 2. Dashed lines are theoretical limits (Fig. 1). field, then superimpose temporal and spatially stochastic events. However, in the absence of such theoretical studies, it is difficult to predict the direction in which scaling relationships would change.

Some degree of caution is needed in relation to both the data and the methodologies. Although the data-set is one of the most detailed ever published, even this is only capable of the examination of pattern over a very restricted range of scales. It can be argued that only scale-invariance over a wide range of scales, say at least orders 2-3 of magnitude (Avnir et al. 1998), would indicate phenomena that transcend the scales of individual population processes. It is possible that the two cases of change in slope in the correlation sum graphs for patchy species are partly the results of the limited size of the study area. For species to be conspicuously patchy within the mapped area, they have to be aggregated on a scale of a few metres. Patches of $O$. pes-caprae and $B$. arvense are in the region of 10 metres in length. As a result, few of these patches occur in the $100 \times 90 \mathrm{~m}$ area

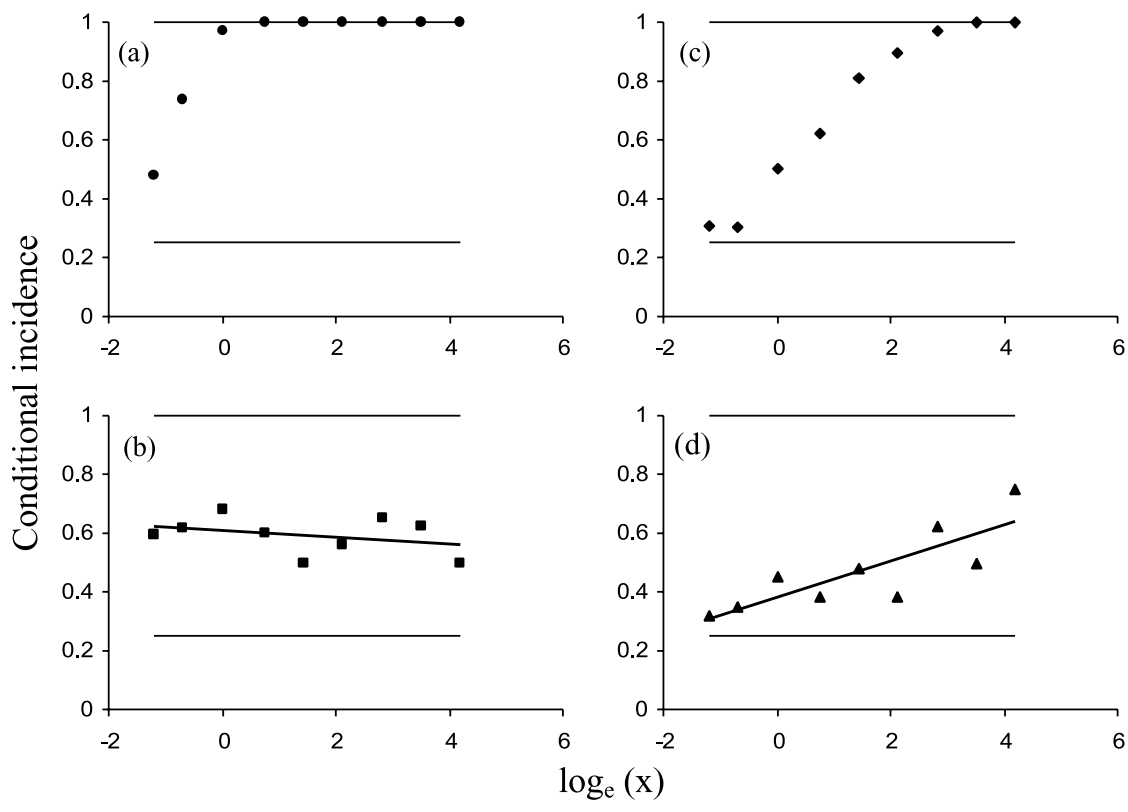

Fig. 7. Conditional incidence for the $100 \times 90 \mathrm{~m}$ area in 1998 ; note that both axes are logged. (a) A. calendula, (b) O. pescaprae, (c) E. botrys, (d) B. arvense. Regression lines are shown for the two species where linear trends appear possible. Dashed lines show the theoretical upper (all plants evenly spread) and lower (all plants in one quadrat) limits. 

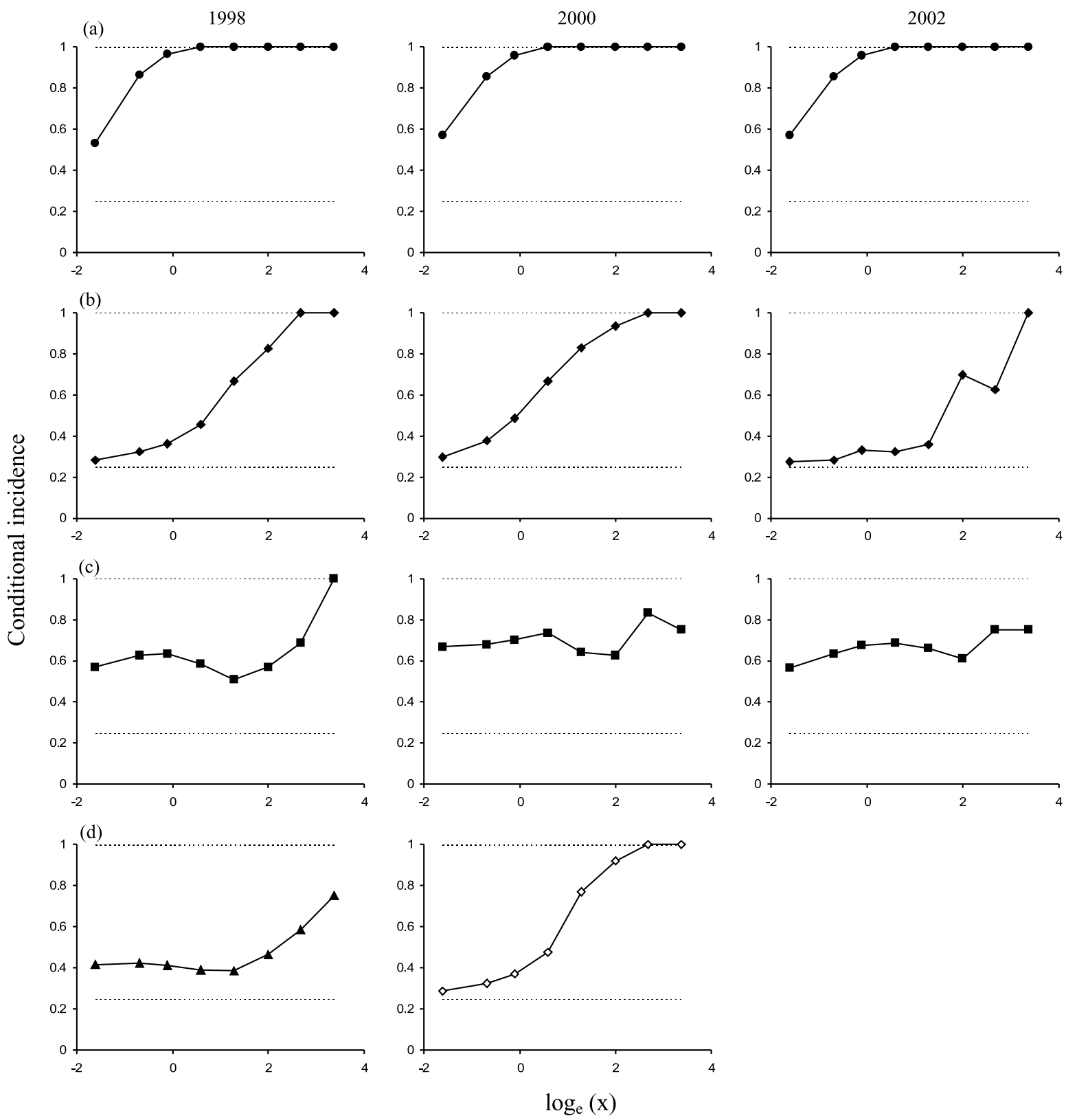

Fig. 8. Conditional Incidence for the $75 \times 45 \mathrm{~m}$ area mapped in all three years: rows indicate (a) A. calendula, (b) O. pes-caprae, (c) $E$. botrys. In row (d) the 1998 graph is for B. arvense, the 2000 graph is for E. cicutarium. Dotted lines show the theoretical upper (all plants evenly spread) and lower (all plants in one quadrat) limits.

of study, and the spatial distribution (degree of aggregation) of the patches themselves cannot be observed. A considerably greater area would be required, increasing the logistical requirements beyond the range of most studies (it is probably not surprising that most studies of scale on spatial pattern are therefore restricted to linear transects). Alternatively, stratified sampling systems could be used.

While $I_{c}$ is related mathematically to $D_{0}$ for selfsimilar patterns by Eq. 2, deviations from self-similarity will be more apparent in $I_{c}$ because the eye is quite sensitive to departures from the horizontal. When a pattern is not self-similar $I_{c}$ can sometimes suggest hypotheses about mechanism, as indicated above. It seems in this way to have an advantage over $\mathrm{D}_{0}$ or $\mathrm{D}_{2}$ estimated from a $\log -\log$ plot, although it is clearly a distinct measure from $\mathrm{D}_{2}$. It should be possible to generate an analogous measure to $I_{c}$ for diversity, by referring local diversity measures to the local incidence, but we do not wish to suggest new methods in this paper. 
All methods provide a global characterization of the plant pattern across the whole of the area studied. Thus, for example, $I_{c}$ aggregates all information about the pattern, i.e. across the centres of dense patches and the diffuse edges around which plants may be widely separated. The value of $I_{c}$ thus depends on the historical development of patches within the field and the extent to which patches have filled the area. If there are different processes acting in different parts of the pattern, these will be confounded by the analytical method. For example, if there is density-dependence in survival rates or reproduction, then the value of $I_{c}$ will depend on the ratio of the area of the diffuse edges to the area occupied by the aggregated centres. Using such "global" analytical methods, rather than "local" methods that treat different areas of the field separately, we must interpret results in terms of broad relationships, reflecting the entire pattern, rather than focussing on subtleties. Thus, the correlation sum will "miss" subtle local events as well.

To this point, we have discussed occurrences of scaleinvariance and reasons why instances of this might be obscured. Scale-invariance, however, is only one of the possible properties of patterns. What about the departures from scale-invariance shown by conditional incidence? It was argued in the Introduction that, in fact, biological processes at characteristic scales may tend to reduce the likelihood of scale-invariance. What types of biological processes might be indicated by the nonfractal shapes of graphs that we have described? For a species that is spreading from new invasion foci, with successful recruitment around parent plants, we would expect that, in parts of the field where it occurs, it would occupy a high proportion of quadrats at a fine scale (i.e. high values of $\mathrm{I}_{\mathrm{c}}$ ). However, in three species (A. calendula, E.botrys and B. arvense), values of $\mathrm{I}_{\mathrm{c}}$ were lowest at fine scales. This is inconsistent with local seed dispersal, and suggests that these species are not spreading rapidly or responding positively to disturbance events. We can therefore suggest, tentatively, that these species may be mostly recruiting from an existing (and therefore long-lived) seed bank and not from recent recruitment events. Few studies have examined the persistence of seeds of these species, but Erodium spp. appear to be long-lived (Rice 1985), while Dunbabin and Cocks (1999) found significant numbers of $A$. calendula seeds remaining in the soil after two years. An absence of recruitment events would, perhaps, not be surprising, in that although these species can sometimes cause significant losses in crop yield, they are primarily plants of pasture, not typical arable weeds. In addition, modern herbicides mean that weeds in many fields only survive to reproductive maturity rarely; most weeds are killed every year. Perhaps, then, low $I_{c}$ at small quadrat sizes may be typical in intensive agricultural systems and less common in organic systems or where weeds have become resistant to herbicides. Thus, the patches of $B$. arvense may be relicts, the remaining plants of a population once more abundant and more widely spread within the field.

The only species with high values of $I_{c}$ at fine scales was $O$. pes-caprae, although this was also scale-invariant according to this method. $O$. pes-caprae had a density profile and patch outline suggestive of a rapidly expanding population. The species reproduces only vegetatively and is believed to disperse mostly through cultivation. However, despite repeated cultivations over the four years of the study and a major increase in average density from 1998 to 2000, the population did not spread appreciably. Increases in population abundance were achieved through increases in density within the area already occupied (i.e. through in-filling of the pattern). The mechanisms of spread in this species are therefore worthy of further investigation. The observed lack of spread is despite the presence of a fringe of isolated individuals surrounding the densest patches, which would normally have been expected to expand rapidly in the absence of competition from conspecifics. Perhaps the dispersing individuals on the fringe may for some reason be specifically poor at over-summering or produce plantlets that for some reason are poorly competitive.

To conclude, this study has provided, for the first time, detailed mapping of various weed species with contrasting ecologies. It has allowed us to examine the generality of scale-invariance in spatial patterns of weed populations. We observed non-trivial scale invariance for species occurring at rather low densities and where species were strongly aggregated, over a wide range of scales, but limited by the size of the study area. The fact that the scale-invariance is not observed for widespread species occurring at higher densities suggests that the scaling is related to critical phenomena that arise if plant populations are kept at the edge of extinction, or equally, at very low densities.

Acknowledgements - We appreciate the cooperation of Dennis Jones in allowing repeated trampling of his crops and Michael Moerkerk for logistical support and encouragement in the project. We also thank Ed Luschei and Andy Rawlinson for their comments on a previous version of the manuscript. RDC thanks Frank Forcella and the staff at USDA-ARS Soil Conservation Laboratory, Morris, Minnesota for their hospitality during data analysis. Special thanks also go to Lisa Crowfoot for production of the maps.

\section{References}

Avnir, D., Biham, O., Lidar, D. et al. 1998. Is the geometry of nature fractal? - Science 279: 39-40.

Bröker, H. M. and Grassberger, P. 1998. SOC in a population model with global control. - Physica A 267: 453-470.

Burrough, P. A. 1983. Multiscale sources of spatial variation in soil. I. The application of fractal concepts to nested levels of soil variation. - J. Soil Sci. 34: 577-597. 
Cousens, R., Doyle, C. J., Cussans, G. W. et al. 1986. Modelling the economics of controlling Avena fatua in winter cereals. - Pestic. Sci. 17: 1-12.

Cousens, R. D., Brown, R., McBratney, A. et al. 2002. Sampling strategy is important for producing weed maps: a case study using kriging. - Weed Sci. 50: 542-546.

Cressie, N. A. C. 1991. Statistics for spatial data. - Wiley.

Dale, M. R. T., Dixon, P., Fortin, M.-J. et al. 2002. Conceptual and mathematical relationships among methods for spatial analysis. - Ecography 25: 558-577.

Dessaint, F., Chadoeuf, R. and Barralis, G. 1991. Spatial pattern analysis of weed seeds in the cultivated soil seed bank. - J. Appl. Ecol. 28: 721-730.

Doyle, C. J., Cousens, R. and Moss, S. R. 1986. A model of the economics of controlling Alopecurus myosuroides in winter wheat. - Crop Prot. 5: 143-150.

Dunbabin, M. T. and Cocks, P. S. 1999. Ecotypic variation for seed dormancy contributes to the success of capeweed (Arctotheca calendula) in Western Australia. - Aust. J. Agric. Sci. 50: 1451-1458.

Dungan, J. L., Perry, J. N., Dale, M. R. T. et al. 2002. A balanced view of scale in spatial statistical analysis. - Ecography 25: 626-640.

Enquist, B. J., Haskell, J. P. and Tiffney, B. H. 2002. General patterns of taxonomic and biomass partitioning in extant and fossil plant communities. - Nature 419: 610-613.

Grassberger, P. 1983. On the critical behaviour of the general epidemic process and dynamical percolation. - Math. Biosci. 63: 157-162.

Grassberger, P. and Procaccia, I. 1983. Characterization of strange attractors. - Phys. Rev. Lett. 50: 346-349.

Haskell, J. P., Ritchie, M. E. and Olff, H. 2002. Fractal geometry predicts varying body size scaling relationships for mammal and bird home ranges. - Nature 418: 527-530.

Hentschel, H. G. E. and Procaccia, I. 1983. The infinite number of generalised dimensions of fractals and strange attractors. - Physica D 8: 435-444.

Howard, C. L., Mortimer, A. M., Gould, P. et al. 1991. The dispersal of weeds-seed movement in arable agriculture. - Proc. Brighton Crop Prot. Conf. -Weeds-1991, pp. 664 673.

Kunin, W. E. 1998. Extrapolating species abundance across spatial scales. - Science 281: 1513-1515.

Kunin, W. E., Hartley, S. and Lennon, J. J. 2000. Scaling down: on the challenge of estimating abundance from occurrence patterns. - Am. Nat. 156: 560-566.

Leduc, A., Prairie, Y. T. and Bergeron, Y. 1994. Fractal dimension estimates of a fragmented landscape: sources of variability. - Landscape Ecol. 9: 279-286.

Levin, S. A. 2003. Complex adaptive systems: exploring the unknown and the unknowable. - Bull. Am. Math. Soc. 40: $3-19$.

Lloyd, M. L. 1967. Mean crowding. - J. Anim. Ecol. 36: 1-30.

Mandelbrot, B. B. 1982. The fractal geometry of nature. - Freeman

\section{Appendix. Calculation of conditional incidence}

In a square area with a side which is a power of two of the minimum quadrat size, conditional incidence $\left(I_{c}\right)$ can easily be calculated by finding the incidence in a series of grids each with half the quadrat size of the previous, and then finding the ratio of incidence at one scale to that at the previous. However, it is often intolerably wasteful to use only field sizes which are a power of 2 of the minimum size (or to throw away
Morisita, M. 1959. Measuring the dispersal of individuals and analysis of the distributional patterns. - Mem. Fac. Sci., Kyushu Univ. Series E (Biol. 2: 215-235.

Morisita, M. 1962. I $\mathrm{I}_{\delta}$-index, a measure of dispersion of individuals. - Res. Popul. Ecol. 4: 1-7.

Pielou, E. C. 1957. The effect of quadrat size on the estimation of the parameters of Neyman's and Thomas's distributions. - J. Ecol. 45: 31-47.

Pielou, E. C. 1977. Mathematical ecology. - Wiley-Interscience.

Rew, L. J. and Cussans, G. W. 1997. Horizontal movement of seeds following tine and plough cultivation: implications for spatial dynamics of weed infestation. - Weed Res. 37: 247256.

Rew, L. J. and Cousens, R. D. 2001. Spatial distribution of weeds in arable crops: are current sampling and analytical methods appropriate? - Weed Res. 41: 1-18.

Rice, K. J. 1985. Responses of Erodium to varying microsites: the role of germination cueing. - Ecology 66: 1651-1657.

Richter, O. and Söndgerath, D. 1990. Parameter estimation in ecology. - VCH Publishers.

Ripley, B. D. 1981. Spatial statistics. - Wiley.

Ross, G. J. S. 1987. MLP: maximum likelihood program. - NAG, Oxford

Sawyer, A. J. 1989. Inconstancy of Taylor's b: simulated sampling with different quadrat sizes and spatial distributions. - Res. Popul. Ecol. 31: 11-24.

Schroeder, M. 1991. Fractals, chaos, power laws: minutes from an infinite paradise. - Freeman.

Shaw, M. W. 1994. Modeling stochastic processes in plant pathology. - Annu. Rev. Phytopath. 34: 523-544.

Shaw, M. W. 1995. Simulation of population expansion and spatial pattern when individual dispersal distributions do not decline exponentially with distance. - Proc. R. Soc. Lond. B 259: 243-248.

Shaw, M. W. 1996. Simulating dispersal of fungal spores by wind, and the resulting patterns. - Aspects Appl. Biol. 46, Modelling in applied biology: spatial aspects, pp. 165172.

Sokal and Rohlf 1995. Biometry. - Freeman.

Sole, R. V., Manrubia, S. C., Benton, M. et al. 1999. Criticality and scaling in evolutionary ecology. - Trends Ecol. Evol. 14: $156-160$.

Southwood, T. R. E. 1966. Ecological methods, with particular reference to the study of insect populations. - Methuen.

Wallinga, J. 1995. The role of space in plant population dynamics: annual weeds as an example. - Oikos 74: 377383.

Wallinga, J., Groeneveld, R. M. W. and Lotz, L. A. P. 1998. Measures that describe weed spatial patterns at different levels of resolution, and their applications for patch spraying of weeds. - Weed Res. 38: 351-359.

Wiles, L. J., Oliver, G. W., York, A. C. et al. 1992. Spatial distribution of broadleaf weeds in North Carolina soybean (Glycine max) fields. - Weed Sci. 40: 554-557.

data in order to make the data set so). The figures in this paper were therefore calculated with a slightly modified algorithm which allows any rectangular field to be used.

The method is recursive. Define the field to be $m \times n$ basic quadrats in size, with $m \geq n$. Initially the field is divided into two parts as close to square as possible by dividing the longer axis into two parts as equally as possible, so these will either both be $\mathrm{m} / 2 \times \mathrm{n}$ or one will be $(m+1) / 2$ and the other $(m-1) / 2$, depending on whether $\mathrm{m}$ is odd or even. At the next stage, this 
procedure is repeated in the resulting rectangles, and the incidence calculated. The sub-division procedure is then repeated within each of the new rectangles, always dividing the longer axis in each rectangle, and so on until it is impossible to continue because each rectangle contains a single grid cell. This leads to estimation of incidence in approximately square quadrats of the correct scale. The conditional incidence is then calculated from the ratio of incidences at successive scales differing by a factor of two. (In fact, incidences can be calculated at each stage of the sub-division, giving area ratios of two. It is best to choose the stage of the subdivision which makes the sides most nearly equal to calculate $I_{c}$, to avoid any influence of anisotropy. To make the graphs shown precise the effective average side of a quadrat can be calculated as the square root of the average area of quadrats.

To clarify this, an example sequence is presented. Starting from a field of $271 \times 156$ basic quadrats, the first two sub-divisions would go:

$$
\begin{aligned}
271 \times 156 & \rightarrow 2 \text { quadrats, } 135 \times 156,136 \times 156 \\
& \rightarrow 4 \text { quadrats, } 135 \times 78,135 \times 78,
\end{aligned}
$$

$$
\begin{aligned}
& 136 \times 78, \quad 136 \times 78 \\
\rightarrow & 2(67 \times 78, \quad 68 \times 78), \quad 4(68 \times 78) .
\end{aligned}
$$

Choose these eight quadrats as being nearly square as possible to start calculating incidences;

$$
\begin{aligned}
& \rightarrow 4(67 \times 39), \quad 12(68 \times 39) \\
& \rightarrow 4(33 \times 39), 28(34 \times 39),
\end{aligned}
$$

again reasonably close to square.

This algorithm always leads to quadrats of reasonably even sides. To see this, first note that the difference between $(\mathrm{m}+1) / 2$ and $(\mathrm{m}-1) / 2$ is unity. Then suppose that $(m+1) / 2$ is even. The next sub-division along this axis will have sides $(m+1) / 4$ in the larger rectangle, and $((\mathrm{m}-1) / 2+1) / 2=\mathrm{m} / 4+1 / 4$ and $((\mathrm{m}-1) / 2-1) / 2=\mathrm{m} / 4-$ $\frac{3}{4}$ in the smaller. Since an exactly analogous argument applies if $(m-1) / 2$ is even, the sides of rectangles in a given direction never differ by more than 1 . Along the long axis, the rule of sub-dividing always in the longer direction means that the most asymmetric quadrats that need ever be used have sides in the ratio $\sqrt{ } 2: 1$, the same as an A4 sheet of paper.

A Pascal program implementing this algorithm is available from MWS on request. 


\section{University Library}

\section{- M M I N E R VA A gateway to Melbourne's research publications}

Minerva Access is the Institutional Repository of The University of Melbourne

Author/s:

Cousens, Roger D.;Wallinga, Jacco;SHAW, MICHAEL

Title:

Are the spatial patterns of weeds scale-invariant?

Date:

2004

Citation:

Cousens, R. D., Wallinga, J., \& Shaw, M. (2004). Are the spatial patterns of weeds scaleinvariant? Oikos, 107(2), 251-264.

Publication Status:

Published

Persistent Link:

http://hdl.handle.net/11343/34647 